\title{
Editorial for the JECR special issue on resistive switching: Oxide materials, mechanisms, and devices
}

\author{
Jennifer L. M. Rupp ${ }^{1,2} \cdot$ Ilia Valov ${ }^{3,4} \cdot$ Daniele Ielmini $^{5}$ \\ Published online: 23 December 2017 \\ (C) Springer Science+Business Media, LLC, part of Springer Nature 2017 \\ Keywords Resistive switching $\cdot$ ReRAM $\cdot$ Ceramics $\cdot$ Electrical properties $\cdot$ Oxides
}

Emulation of neural networks by redox-based Resistive Random Access Memories (ReRAMs) with components such as thin films of ceramic materials are considered by the technological roadmap (ITRS) as a promising concept for the next generation non-volatile memory storage and as an important key towards computation with neuromorphic algorithms. ReRAMs are regarded as conceptually new building units in modern nanoelectronics, finding application not only as a memory, but also as selectors, for logic operations and neuromorphic computing circuits beyond the von Neumann concept, being capable of bio-inspired cognitive functions, such as machine learning and pattern recognition. The information is saved in ReRAMs as particular resistances of the devices adjustable by voltage stimuli, where in the most simple case the high resistive state represents the Boolean 0 and the low resistive state - the Boolean 1. The devices show outstanding potential for scaling down to the atomic level, integration, low-power consumption, sub-nanosecond operation time range and digital and/or analog volatile and/or non-volatile information storage. In these devices, the switching relies on redox

Jennifer L. M. Rupp

jrupp@mit.edu

1 Department of Materials Science and Engineering, Massachusetts Institute of Technology, Cambridge, MA 02139, UK

2 Department of Electrical Engineering and Computer Science, Massachusetts Institute of Technology, Cambridge, MA 02139, UK

3 Forschungszentrum Jülich, Electronic Materials (PGI 7), Wilhelm-Johnen-Str, 52425 Jülich, Germany

4 Institute of Materials in Electrical Engineering and Information Technology 2, RWTH Aachen University, Sommerfeldstr. 24, 52070 Aachen, Germany

5 Dipartimento di Elettronica, Informazione e Bioingegneria, |Politecnico di Milano, Milan, Italy reactions and mixed ionic-electronic transport at the nanoscale, in cells/devices of the type MEM (metal-electrolytemetal) or MIM (metal-insulator-metal) where oxides and higher chalcogenides are typically used as ion conducting materials. ReRAM devices are operated at extremely harsh conditions characterized by high electric fields (up to $10^{8} \mathrm{~V} / \mathrm{m}$ ) and extremely high current densities in the range above $\mathrm{MA} / \mathrm{cm}^{2}$ and show perspective for future memory and neuromorphic computing devices.

In this special issue of the Journal of Electroceramics, we present a selection of invited and contributed articles, which focus on both materials and device aspects. These papers span a wide range reviewing and proposing materials for the switching oxides, including investigation of the switching mechanism, highlighting the involved nanionic processes and highlighting novel operation principles. We are fortunate to have attracted 16 high quality and timely contributions to this issue composed of invited reviews highlighting either material, probing or device aspects on the theme of this special issue.

An invited review on resistive switching devices and their applications as mixed signal analog-digital neuromorphic computing architectures and other application fields by Zidan, Chen, Indiveri and Lu leads off the special issue [1]. In this paper, the development and opportunities of memristive switching devices for replacing classic binary transistors and potential computing architectures are discussed by the authors. Resistive switches offer the intrinsic ability of ready processing in 3D memory array architectures with exciting potential for mass storage applications and handling of big data. In a joint contribution lead by Chen et al. colleagues from Stanford, GigaDevice Semiconductor Inc., IMM-CNR, National Chiao Tung University, Chinese Academy of Science and Minatec Campus Grenoble came together to present recent advances from materials and devices, to 3D integration and bottom up ReRAM fabrication [2]. A general overview on modeling resistive switching across scales ranging from electrons to device 
level to elucidate best architectures for resistive switching materials is given by Ambroio, Magyari-Köpe, Onofrio, Islam, Duncan, Nishi and Strachan [3]. Following this work, Riess discusses conditions for resistive switching in oxide conductors including the effects of humidity and protons in ambient [4]. Yang, Takahashi, Tsurumaki-Fukuchi, Arita, Moors, Buckwell, Mehonic and Keynon turn to the atomistic scale and review in situ TEM and STM probing characterization techniques of conducting filaments in memristive devices [5]. Special emphasis on conductive atomic force microscopy (c-AFM) as a tool to capture resistive switching processes in oxides is debated by Lanza, Celano and Miao [6]. Turning to understanding and designing oxide architectures Chen, Tappertzhofen, Barnaby and Kozicki explore the virtues of $\mathrm{SiO}_{2}$ based conductive bridging random access memories and discuss insight into their memory array and neuromorphic computing applications [7]. Lian, Wang, Yan, Yang and Miao newly propose a criteria for selecting high-performance memristor materials based on the statistical results and the temperature evolution of conductive filaments with examples of $\mathrm{TaO}_{\mathrm{x}}, \mathrm{HfO}_{2}$ and $\mathrm{NiO}$ as resistive switching materials [8]. Hafnium oxide, an important memory material in the electronics industry, is studied in its oxygen ionic migration and switching characteristics by Thammasack, De Micheli and Gaillardon [9]. The role of Ti interlayers on the switching dynamics is the focus of their work. To allow a high versatility of ReRAM architectures, the mode of operation as either a 2- or 3-terminal device is explored, with implications for oxide nanostructures and switching characteristics presented by Tsuuoka, Hasegawa, Terabe and Aono [10]. Following this work, the team of Burriel with Bagdzevicius, Maas and Boudard reviews recent progress on perovskite materials as resistive switching oxides [11]. Here, it is the choice of the cations that defines the overall mixed ionic electronic transport and redox-processes of the material class, where careful guidelines on structure, chemistry and doping are presented for their use as resistive switches. The role of metal-insulator transitions on resistive switching of perovskite oxides and potential impact on structural and chemical design rules are discussed by Gonzales-Rosillo and co-workers from the Puig group [12]. Kubicek, Taibl, Navickas, Hutter, Fafilek and Fleig investigate and review bias effects and mechanisms at high and low temperature for strontium titanate perovskites [13]. Yang and the Guo team explore how strontium titanate can be employed as resistive switch to emulate neurological and psychological functions [14]. Raeis-Hosseini and Lee review resistive switching memories using biomaterials based on flexible nanoelectronics [15]. Finally, optical memristive switches are discussed by Koch, Hossbacher, Emboras and Leuthold [16].

Concluding the guest editors highlight the fact that the property of oxide-ceramics employed as resistive switches contributes to new discoveries on material behavior at high local electric fields, phases, structures and electrochemistry. In an utopic version, these are the keys to fast data transfer, computation and high capacity information storage and define the future of nanoelectronics and information technology. The special issue shows that the field is excitingly crossing boundaries where material science, electrical engineering, computer science, electrochemistry and neurological science unite to tackle new ways to compute beyond classic binary transistors, dominant for decades. One may see this as a contribution to the Fourth Industrial Revolution for which simple material structures and easy implementation of switching devices may have fast and immediate impact on society.

Acknowledgements All authors and contributors are thanked by the guest editors for their high quality work, critical discussion and sharing of scientific concepts and discoveries through this special issue. The editors were especially thankful to the open attitude of authors coming together from various teams around the world to provide unique review articles on selected hot topics. Finally, we thank the chief editor Prof. Harry Tuller for his enthusiasm and dedication to the field of Electroceramics.

\section{References}

1. M.A. Zidan, A. Chen, G. Indiveri, W.D. Lu, Memristive computing devices and applications. J. Electroceram. (2017). https://doi.org/ 10.1007/s10832-017-0103-0

2. H.-Y. Chen et al., Resistive Random Access Memory (RRAM) Technology: From Material, Device, Selector, 3D Integration to Bottom-up Fabrication. J. Electroceram. (2017). https://doi.org/10. 1007/s10832-017-0095-9

3. S. Ambrogio et al., Modeling resistive switching materials and devices across scales. J. Electroceram. (2017). https://doi.org/10. 1007/s10832-017-0093-y

4. I. Riess, Review of mechanisms proposed for redox based resistive switching structures. J. Electroceram. (2017). https://doi.org/10. 1007/s10832-017-0092-Z

5. Y. Yang et al., Probing electrochemistry at the nanoscale: in situ TEM and STM characterizations of conducting filaments in memristive devices. J. Electroceram. (2016). https://doi.org/10. 1007/s10832-017-0069-y

6. M. Lanza, U. Celano, F. Miao, Nanoscale characterization of resistive switching using advanced conductive atomic force microscopy based setups. J. Electroceram. (2017). https://doi.org/10.1007/ s10832-017-0082-1

7. W. Chen et al., $\mathrm{SiO}_{2}$ based conductive bridging random access memory. J. Electroceram. (2017). https://doi.org/10.1007/s10832-017-0070-5

8. X. Lian, M. Wang, P. Yan, J.J. Yang, F. Miao, Reset switching statistics of $\mathrm{TaO}_{\mathrm{x}}$-based Memristor. J Electroceram (2016). https:// doi.org/10.1007/s10832-017-0094-x

9. M. Thammasack, G. De Micheli, P.-E. Gaillardon, Effect of $\mathrm{O}^{2-}$ migration in $\mathrm{Pt} / \mathrm{HfO}_{2} / \mathrm{Ti} / \mathrm{Pt}$ structure. J. Electroceram. (2017). https://doi. org/10.1007/s10832-017-0077-y

10. T. Tsuruoka, T. Hasegawa, K. Terabe, M. Aono, Operating mechanism and resistive switching characteristics of two- and threeterminal atomic switches using a thin metal oxide layer. J Electroceram (2016). https://doi.org/10.1007/s10832-016-0063-9 
11. S. Bagdzevicius, K. Maas, M. Boudard, M. Burriel, Interface-type resistive switching in perovskite materials. J. Electroceram. (2017). https://doi.org/10.1007/s10832-017-0087-9

12. J.C. Gonzalez-Rosillo et al., Volume Resistive Switching in metallic perovskite oxides driven by the Metal-Insulator Transition. J. Electroceram. (2017). https://doi.org/10.1007/s10832-017-0101-2

13. M. Kubicek et al., Resistive states in strontium titanate thin films: Bias effects and mechanisms at high and low temperature. J. Electroceram. (2017). https://doi.org/10.1007/s10832017-0081-2
14. X.-B. Yin, Z.-H. Tan, R. Yan, X. Guo, Single crystalline $\mathrm{SrTiO}_{3}$ as memristive model system: From materials science to neurological and psychological functions. J. Electroceram. (2017). https://doi. org/10.1007/s10832-017-0083-0

15. N. Raeis-Hosseini, J.-S. Lee, Resistive switching memory using biomaterials. J. Electroceram. (2017). https://doi.org/10.1007/ s10832-017-0104-Z

16. U. Koch, C. Hoessbacher, A. Emboras, J. Leuthold, Optical Memristive switches. J. Electroceram. (2017). https://doi.org/10. 1007/s10832-017-0072-3 